\title{
Quantitative trait loci for fluctuating asymmetry of discrete skeletal characters in mice
}

\author{
LARRY J . LEAMY*†, ERIC J . ROUTMAN\$ \& J AMES M. CHEVERUD§ \\ $\dagger$ Department of Biology, University of North Carolina at Charlotte, Charlotte, North Carolina 28223, U.S.A., \\ ‡Department of Biology, San Francisco State University, San Francisco, California 94132, U.S.A. and §Department \\ of Anatomy and Neurobiology, Washington University School of Medicine, St. Louis, Missouri 63110, U.S.A.
}

\begin{abstract}
Levels of fluctuating asymmetry (FA) are often taken as indicators of the degree to which genotypes differ in their ability to buffer genetic and environmental sources of variation. Interval mapping techniques were used to search for quantitative trait loci (QTLs) affecting directional asymmetry (DA) and fluctuating asymmetry (FA) in six bilateral discrete skeletal traits in house mice. These six characters as well as 76 microsatellite markers were scored in over 500 mice that resulted from crosses of $F_{1}$ mice originally produced from matings of the Large (LG/J) and Small (SM/J) inbred strains. The number of QTLs affecting DA in each of the characters was no more than expected by chance alone so it was concluded that there was little evidence for individual genes affecting DA. There appeared to be a genetical basis for FA in these characters, however, because the number of QTLs significantly affecting FA (10 at the $5 \%$ level, three at the 1\% level) was greater than expected by chance alone. The 10 QTLs significantly affecting FA in any given character were located on eight different chromosomes, mostly at locations for QTLs affecting other characters or DA in other characters. Their cumulative contribution to the total phenotypic variance was small, averaging only $3.9 \%$ per locus. Dominance genotypic values for these QTLs were more extreme than additive genotypic values, suggesting that heterozygotes at many loci are better buffered than homozygotes and that allelic interactions (dominance) may play an important role in the production of FA.
\end{abstract}

Keywords: directional asymmetry, fluctuating asymmetry, mice, quantitative trait loci, skeletal traits.

\section{Introduction}

Does nondirectional variation in the differences between right and left sides of bilateral characters, normally referred to as fluctuating asymmetry (FA), have a genetic basis? Conventional wisdom suggests that the same genes govern both sides of these kinds of characters and that few, if any, genes act singly or epistatically on the separate sides (Palmer, 1994; Leamy, 1997a). Because of this, differences between sides that constitute FA might be expected to exhibit little or no genetical variation, and in fact most heritability estimates of FA have been low or zero (see Leamy, 1997a) although occasional others (Thornhill \& Sauer, 1992) have been high. Perhaps more importantly, FA levels often significantly differ between heterozygotes and homozygotes (see

\footnotetext{
*Correspondence. E-mail: ljleamy@email.uncc.edu
}

Leamy, 1984), suggesting that nonadditive genetic effects may play an important role in FA.

Even though the genetical/environmental basis for FA is far from clear, FA generally continues to be viewed as solely environmentally determined, reflecting random noise processes that render it among the most useful measures we have of developmental stability (Zakharov, 1994). The level of developmental stability attained by organisms in the face of environmental challenges, however, may well be genetically based. Whatever the actual basis of FA, it has been used in a number of studies to compare developmental stability levels in populations subjected to various genetical or environmental stressors such as inbreeding, hybridization, parasitic infections, pollution and sexual selection (Palmer et al., 1994; Zakharov, 1994). In contrast to FA, directional asymmetry (DA), another form of bilateral asymmetry in which one side is consistently larger 
than the other, has not generally been advocated as a measure of developmental stability because of its presumed genetical basis (Palmer, 1994).

In recent years, interval mapping methods (Lander \& Botstein, 1989; Dietrich et al., 1996) have been developed which allow the identification and mapping of quantitative trait loci (QTLs) to specific sites between molecular markers on each chromosome. These methods also allow tests for additivity and dominance of the QTLs, and thus seem to offer great potential for our understanding of the genetical basis of asymmetries in bilateral characters. In a previous investigation using this approach to analyse asymmetry, we (Leamy et al., 1997) discovered a significant number of QTLs affecting DA, but not $\mathrm{FA}$, in a suite of 10 mandibular dimensions in mice. In this paper we report the results of a follow-up QTL analysis using these same mice, but assessing DA and FA in six discrete skeletal characters (primarily the presence/absence of skull foramina). We had assumed a priori that we might expect the same result as before, but in fact found the opposite: significant numbers of QTLs for FA, but not DA, in these characters.

\section{Materials and methods}

\section{The population and characters}

The house mice used in this study were produced from crossing $F_{1}$ hybrids between the Large (LG/J) and Small $(\mathrm{SM} / \mathrm{J})$ inbred strains obtained from the Jackson Laboratory. Originally, the LG/J strain was selected for large size (Goodale, 1941) and the SM/J strain was selected for small size (MacArthur, 1944); but when received by the Jackson Laboratory, selection was abandoned and both strains were inbred. Using the $\mathrm{LG} / \mathrm{J}$ and $\mathrm{SM} / \mathrm{J}$ strains, Cheverud et al. (1996) found between seven and 17 QTLs, located on 16 of the 19 autosomes, affecting body weight at various ages.

DNA was extracted from the spleens of mice in both the Large and Small strains (see Routman \& Cheverud, 1995 for the protocol). Then these strains were screened for a total of 472 microsatellite loci using PCR amplification techniques modified from Dietrich et al. (1996). The amplification products were visualized with the use of ethidium bromide staining on 5-6\% agarose gels (Routman \& Cheverud, 1995). Nearly one-half (47\%) of the loci were found to be polymorphic between the two strains (Routman \& Cheverud, 1995), and of these, 76 that mapped in representative areas on the 19 autosomes were chosen for scoring in the $F_{2}$ mice
(Fig. 1). The $\mathrm{X}$ chromosome was not included in this study because of its low incidence of polymorphic microsatellite loci (Routman \& Cheverud, 1995).

After this preliminary screening of microsatellites, 10 single-pair matings of Large mice (females) $\times$ Small mice (males) were made, which produced a total of $41 \mathrm{~F}_{1}$ hybrid mice. The $\mathrm{F}_{1}$ mice in turn were single-pair mated by 70 days of age, and eventually produced a total of $535 \mathrm{~F}_{2}$ mice. After 21 days of age, the $F_{2}$ litters were weaned and sexes were caged separately. All $\mathrm{F}_{2}$ mice were killed at 70 days of age, their spleens removed, and their skeletons prepared by exposure to dermestid beetles.

Scoring of the 76 microsatellite loci was accomplished following the same procedure as already outlined for the Large and Small strains. Some lanes were not resolvable on the gels, so the loci varied in their total sample sizes (see Leamy et al., 1997). In addition, one locus, D10Mit20, was used only as a dominant marker as the heterozygote could not be distinguished from the SM/J homozygote. The positions of the 76 microsatellite loci based on recombination percentages in the $\mathrm{F}_{2}$ mice themselves derived from the MAPMAKER 3.0B program (Lander et al., 1987; Lincoln et al., 1992) have previously been given (Cheverud et al., 1996; Leamy et al., 1997) and are shown as a genetic map in Fig. 1. A total of $1500 \mathrm{cM}$ was defined by these 76 loci, as well as 55 intervals with an average interval length of $27.5 \mathrm{cM}$.

Six bilateral discrete skeletal characters that were polymorphic in the $\mathrm{F}_{2}$ mice were chosen for analysis. These characters, abbreviated $\mathrm{C} 1-\mathrm{C} 6$, are: $\mathrm{C} 1$, maxillary foramen I; C2, maxillary foramen II; C3, foramen palatinus minus posterius; $\mathrm{C} 4$, foramen pterygoideus double; C5, preoptic sutures; C6, metoptic roots abnormal. For descriptions of C1-C4, see Berry (1963), and for C5 and C6, see Truslove (1954). All characters were located on the skulls of the mice, and four of the six involved the presence or absence of various foramina. These characters were scored as either present (1) or absent (0) on both left and right sides. All mice were scored once because of the labour involved, but scoring for each of the characters was repeated for a random sample of 30 mice and it was discovered that the repeatability was good, averaging over $90 \%$ agreement for all characters and their asymmetries.

\section{Statistical analysis}

For each of the six bilateral characters, DA was assessed by the signed differences between sides (Palmer, 1994). Thus possible DA values for any 
character were $-1,0$, and +1 . In addition, these values were summed over all characters (maximum value range from -6 to +6 ) to yield a character (DASUM) that was useful as an overall indicator of DA. FA was defined by the unsigned or absolute differences between sides (Palmer, 1994; Leamy, $1997 \mathrm{a}$ ), so that possible values were 0 for symmetric characters (those with the character absent on both left and right sides or present on both sides: $0-0$ or 1-1) and 1 for asymmetric characters (those with the character present on one side, but absent on the other: $1-0$ or $0-1)$. A composite character for FA (FASUM) was obtained by adding all six individual FA scores for each of the characters (possible range from 0 to 6). Finally, it was convenient to define a measure for each of the six bilateral characters themselves rather than just their asymmetries. Each bilateral character therefore was defined as present (1) if either left or right side exhibited that character, but absent (0) otherwise. It was then possible to search for any QTLs significantly affecting these characters (see below), and if found, to determine whether they were the same as or different from those affecting DA or FA in each of these characters.

For each of the six bilateral characters, DA was tested for significance by the McNemar test for significance of changes (Sokal \& Rohlf, 1995; Leamy, 1997b). This test is particularly appropriate here because it is the attribute data equivalent of a randomized block design in which there are two treatments (Sokal \& Rohlf, 1995). All probabilities generated in these tests were evaluated for significance by the sequential Bonferroni procedure (Rice, 1989). Any result reaching significance for a given character would imply that the incidence of that character is greater on the left side than on the right side or vice versa, and thus that DA exists. In addition, the distribution of DASUM was tested for skewness and kurtosis to rule out the presence of antisymmetry (AS), another form of bilateral asymmetry where there is a statistically significant difference between sides but the side that is larger varies at random (Palmer, 1994). Antisymmetry typically is indicated by a significant, negative kurtosis statistic (Palmer \& Strobeck, 1992).
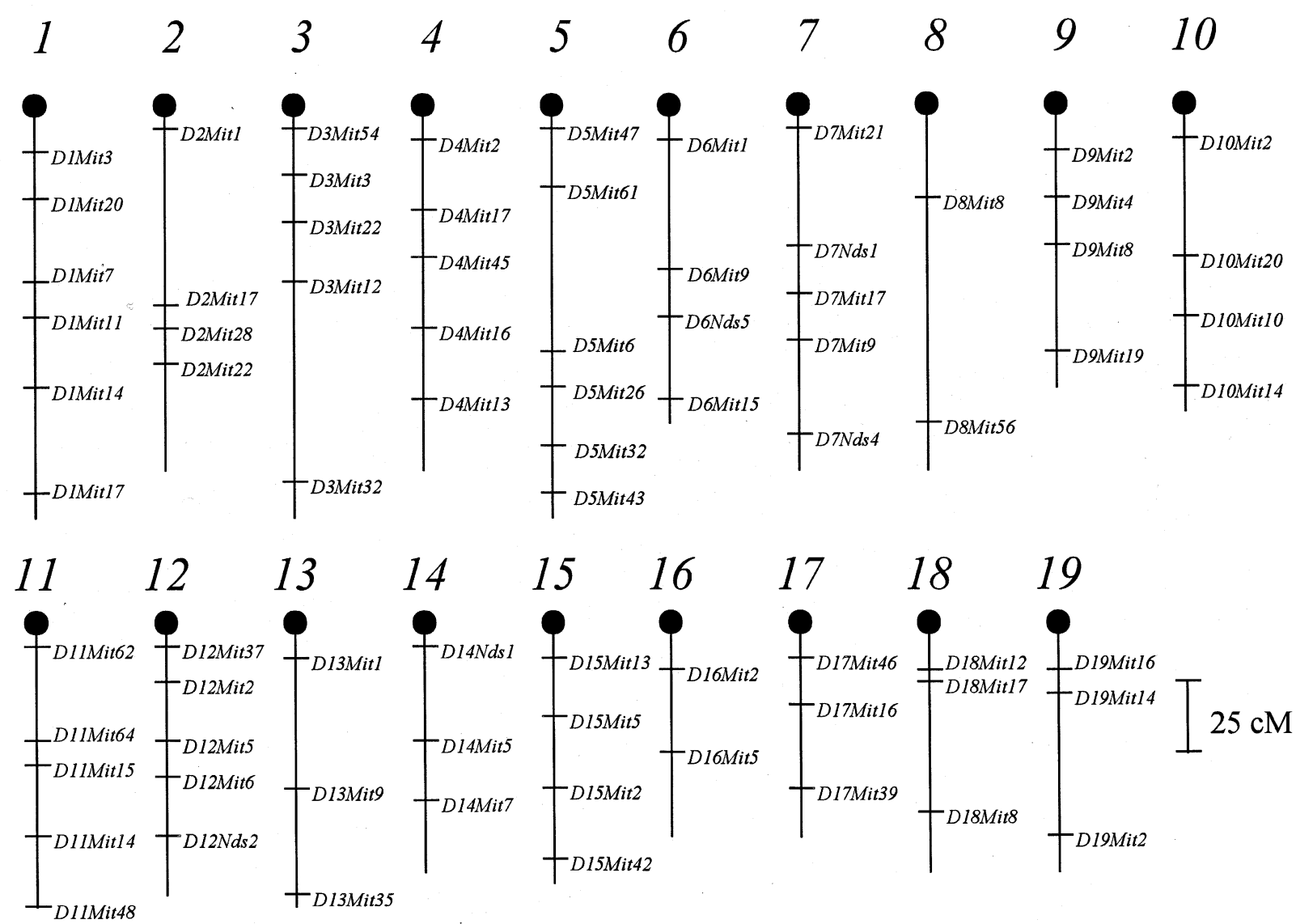

Fig. 1 Relative positions of microsatellite markers scored in the $F_{2}$ mice from an original cross of LG/J with SM/J. 
Logistic regression (Sokal \& Rohlf, 1995) was used to test simultaneously for the significance of sex, dam (differences among the progeny from different $F_{1}$ dams), block (differences among the five periods of rearing), litter size effects, and all interactions between these four factors, on DA and FA in each of the six bilateral characters. All probabilities generated from the regression analyses were assessed via the sequential Bonferroni test (Rice, 1989) where there were considered to be 12 separate tests (DA and FA characters combined) for each of these four factors. Results of these tests were nonsignificant for all characters, so no adjustments were made. Similarly, results of multiple regression of each of these four factors on both DASUM and FASUM also were not significant, so the QTL analysis described below was conducted on these composite characters as well with no original adjustments made for any of these factors.

\section{QTL analyses}

A search for QTLs affecting the six discrete characters and their signed (DA) and unsigned differences between sides (FA), was carried out with the MAPMAKER/QTL 1.1b program (Lander et al., 1987; Lincoln et al., 1992). This program makes use of a maximum likelihood model that provides estimates of additive $(a)$ and dominance genotypic values $(d)$ and the percentage of effect for putative QTLs in every $2 \mathrm{cM}$ interval between adjacent molecular markers. It also calculates a LOD score for each interval, where this score represents a ratio of the $\log _{10}$ likelihood that a QTL exists to the $\log _{10}$ likelihood that it does not exist in that interval. Thus LOD scores are highest where the existence of a QTL is most probable and therefore were used to indicate the probability of QTLs being present. In applying the MAPMAKER program, no adjustment was made for markers on other chromosomes (Zeng, 1994) because Cheverud et al. (1996) obtained very little increase in statistical power when they made such adjustments in their previous QTL analysis on these same mice, and because this procedure complicates data analysis when there are missing data.

The large number of LOD scores calculated with so many chromosomes and characters used in this QTL analysis clearly dictated that the significance level for any single score needed to be adjusted. Cheverud et al. (1996) previously determined the appropriate critical values for significance on the basis of the distribution of LOD scores obtained in
QTL analyses run on 500 samples of random, normally distributed traits $[\mathrm{N}(0,1)]$ obtained from simulations. For each of the 19 chromosomes, those LOD scores obtained in the simulations 5 and $1 \%$ of the time, respectively, were used as the critical values for the $5 \%$ and $1 \%$ significance levels (see Cheverud et al., 1996). These critical values were used for the LOD scores generated for the composite asymmetry characters DASUM and FASUM. Thus if the LOD score for any of these characters equaled or exceeded the appropriate critical value for that particular chromosome, the null hypothesis of no QTL effect was rejected. However, it was not certain whether these previously generated critical values would be appropriate for determining significance of the LOD scores for the individual characters and their asymmetries given the limitations of their distributions ( 0 and 1 values only). Therefore new critical values were established for use with these characters by the method described above but using 500 random samples of size 530, each generated with 0 and 1 values only where the overall incidence of $1 \mathrm{~s}$ was 0.2 .

In applying the MAPMAKER program to all characters, a single QTL model that included both additive and dominance genotypic effects first was fitted to the data. If statistically significant results were obtained as explained above, the patterns of LOD scores and additive/dominance effects were examined to determine whether a second QTL might be present on the chromosome. Abrupt changes in the sign of additive/dominance effects or bimodal distributions of LOD scores are two kinds of patterns that often suggest the possibility of two QTLs (Cheverud et al., 1996), although linked QTLs do not always exhibit these patterns (Haley \& Knott, 1992). If a second QTL seemed likely for any character and chromosome, a two-QTL model was employed. Significance for such two-QTL models was indicated when there was a statistically significant improvement in fit (using the $\chi^{2}$-statistic) over the one-QTL model.

For all significant QTLs, confidence intervals were determined as the distances in $\mathrm{cM}$ on either side of the QTL locations where there was a drop in the peak LOD score of 1.0. The confidence limits determined in this manner describe positions 10 times less likely than the peak position to represent a QTL. In some cases these confidence intervals are not very useful because this drop is not reached even at the location of the proximal or distal molecular marker, but these intervals do at least provide a rough idea of the potential boundaries of the QTLs.

(C) The Genetical Society of Great Britain, Heredity, 80, 509-518. 
In addition, for each significant QTL, additive and dominance genotypic effects were tested for significance using $\chi_{1}^{2}$ with conventional critical values $(3.84$ for $5 \%$ and 6.64 for $1 \%$ ). Standard errors also were calculated for the $a$ - and $d$-values by dividing them by the square root of the chi-squared values (equivalent to Student's $t$-values) obtained in the additive/ dominance tests.

Conclusions about the overall presence/absence of QTLs affecting DA and FA in the six discrete skeletal characters were based on the total number of QTLs expected to be significant by chance alone. For the six DA (or six FA) characters, this number was assumed to be six characters $\times 19$ chromosomes $\times 0.05=5.7$ at the $5 \%$ level or $6 \times 19 \times 0.01=1.1$ at the $1 \%$ level. Thus if the number of QTLs significantly $(P<0.05)$ affecting DA or FA in the six characters exceeded six (or exceeded one if significant at the $1 \%$ level), this was more than expected by chance alone and it was concluded that at least some of these QTLs were genuine. For either the DASUM or the FASUM character, the number of QTLs expected by chance was $1 \times 19 \times 0.05=0.95 \quad(5 \% \quad$ level $) \quad$ and $1 \times 19 \times 0.01=0.19(1 \%$ level $)$.

These probability arguments depend upon DA and FA in each of the six characters being independent and in fact pairwise Pearson correlations (equivalent to phi coefficients) of these values showed that this generally was true. Thus only one of the 15 pairwise correlations of DA (and overall) values, and none of the 15 correlations of FA values for each of the six characters, was significantly greater than zero when evaluated with the sequential Bonferroni procedure (Rice, 1989).

\section{Results}

Table 1 gives the frequencies for the overall characters (present on either side), for the left and right sides, and for the signed and unsigned differences for each of the six bilateral characters. A few mouse skulls were broken, and some characters were ambiguous and therefore not used, so the total number of mice available for each character varied (from 489 to 516). As may be seen, C3 (foramen palatinum minus posterius) is most frequent, and $\mathrm{C} 6$ (metoptic roots abnormal) least frequent, in this population of mice. McNemar tests show that the frequencies of the left and right sides are similar for all characters except that for C6 which exhibits significant DA (left side more frequent than right side; $P<0.01)$. Characters $\mathrm{C} 1-\mathrm{C} 5$ as well as the sum of all six characters (SUM) exhibit classical FA, with their mean DA values all being close to zero. Although not shown in this table, skewness (0.01) and kurtosis (0.36) statistics calculated for DASUM are not significant $(P>0.05)$, suggesting that this character shows no trace of antisymmetry and thus is an acceptable indicator of overall DA.

New 5\% critical cutpoints for determining significance of the LOD scores, generated as previously explained, varied from 1.615 (chromosome 16) to 2.255 (chromosome 1). Their mean was 1.972 , nearly identical with that of 1.920 previously calculated by Cheverud et al. (1996). Further, the Pearson correlation between the two sets of $5 \%$ critical cutpoints was $0.74(P<0.01)$, indicating a high degree of association. The $1 \%$ critical values generated for the $0 / 1$ values ranged from 2.363 (chromosome 16) to 3.133 (chromosome 4) with an average (2.804) that was somewhat higher than that of 2.620 calculated

Table 1 Mean frequencies for each of the discrete mouse skeletal characters (overall $=$ present on either side) and for the left and right sides and the signed (DA) and unsigned differences between sides (FA) for the bilateral characters and their sums. $N$, sample size. $P$, probability from the McNemar test of significance of the differences between sides

\begin{tabular}{lccccccc}
\hline & $\mathrm{N}$ & Overall & Left side & Right side & $P$ & Mean DA & Mean FA \\
\hline C1 & 489 & 0.626 & 0.429 & 0.446 & 0.555 & 0.016 & 0.376 \\
C2 & 516 & 0.787 & 0.560 & 0.560 & 1.000 & 0 & 0.453 \\
C3 & 510 & 0.947 & 0.835 & 0.802 & 0.137 & -0.033 & 0.257 \\
C4 & 501 & 0.876 & 0.715 & 0.737 & 0.371 & 0.022 & 0.301 \\
C5 & 484 & 0.318 & 0.225 & 0.227 & 0.916 & 0.002 & 0.183 \\
C6 & 502 & 0.203 & 0.171 & 0.084 & $0.001^{* *}$ & -0.088 & 0.151 \\
Sum & 391 & 2.927 & 2.949 & 2.905 & 0.491 & -0.043 & 1.706 \\
\hline
\end{tabular}


by Cheverud et al. (1996). The correlation between these two sets of values was $0.48(P<0.05)$, somewhat lower than that for the $5 \%$ values, presumably because this correlation is estimated from a very small sample in the simulation (five scores only).

Results of interval mapping first for the overall characters themselves $(\mathrm{C} 1-\mathrm{C} 6)$ are shown in the Appendix. Locations of putative QTLs are given by the number of $\mathrm{cM}$ from the proximal flanking molecular marker on each of the chromosomes and are depicted in Fig. 2. Confidence intervals also are given for the QTLs, although these were unresolved at either the proximal or distal limit for the QTLs on chromosome 8 , apparently because of a relative lack of genotypic variability in the middle of the interval, combined with the $0 / 1$ nature of the data. Also, in some cases these intervals extend past the ends of the most proximal or distal molecular markers.

As may be seen, 18 QTLs significantly affecting the skeletal characters are located on 10 of the 19 autosomes. Fully half (nine) of the LOD scores reach significance at the $1 \%$ level, this being far greater than the 1.1 expected by chance alone. The distribution of QTLs affecting each of the characters is: $\mathrm{C} 1,3 ; \mathrm{C} 2,3 ; \mathrm{C} 3,0 ; \mathrm{C} 4,2 ; \mathrm{C} 5,7 ; \mathrm{C} 6,3$; so foramen palatinus minus posterior (C3) is least affected whereas preoptic sutures (C5) is most affected by these QTLs. Analysis of the confidence intervals suggests that there is probably just one QTL on each of chromosomes 2, 4, 7 and 8 that is affecting more than one skeletal character, whereas chromosome 11 appears to have two QTLs (with nonoverlapping confidence intervals), one affecting $\mathrm{C} 2$ and $\mathrm{C} 5$ and another affecting C4. If so, the total number of significant QTLs affecting these skeletal characters is 11 . The average percentage of effect of these QTLs is $3.7 \%$, and their average absolute additive and dominance genotype values are similar: $0.081 \pm 0.011$ and $0.097 \pm 0.011$.

Table 2 provides descriptive data for all putative QTLs significantly affecting DA in each of the six characters and over all characters (DASUM). Locations are given as before by the number of $\mathrm{cM}$ beyond the proximal flanking marker and also are depicted in Fig. 2. QTLs reaching significance for the DA characters are located on eight of the 19 autosomes. Two QTLs on chromosome 17 affect DA in the maxillary foramen II character $(\mathrm{C} 2)$, for which only one joint LOD score and percentage of effect is generated by the MAPMAKER program. In general, these QTLs for DA do not appear to be at the same loci as those QTLs affecting the particular characters themselves (see Fig. 2). One QTL on chromosome 13 significantly affects overall DA (DASUM), although this apparently is attributable to DA3 because QTL runs of the sum of all DA characters except DA3 did not reach significance. The QTLs exhibit a mean percentage of effect of $4.1 \%$, a value very comparable to that calculated above for the overall characters. The average absolute additive and dominance genotypic values for these QTLs (excluding that for DASUM) are not significantly different $(P>0.05): 0.100 \pm 0.018$ and $0.156 \pm 0.036$.

Nine QTLs affecting DA reach significance at the $5 \%$ level (Table 2), a value which is somewhat
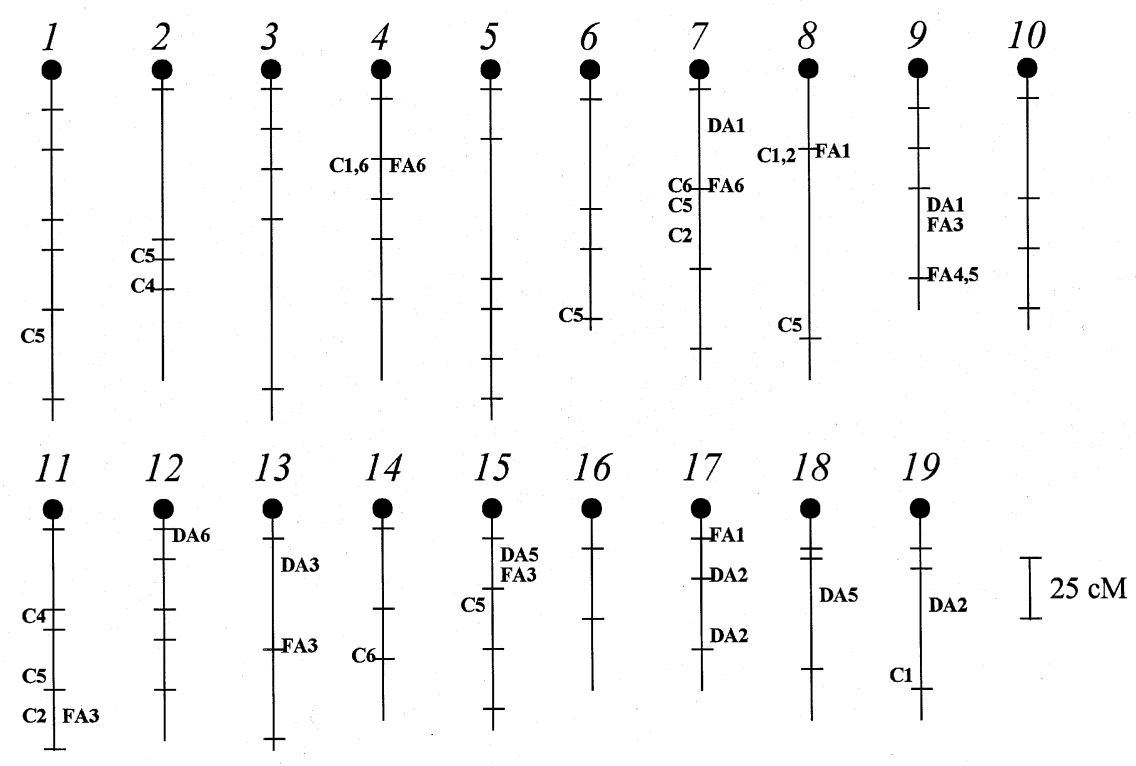

Fig. 2 Relative positions of QTLs significant for the six discrete skeletal characters in mice $(\mathrm{C} 1-\mathrm{C} 6)$ as well as directional asymmetry (DA) and fluctuating asymmetry (FA) in these characters.

(C) The Genetical Society of Great Britain, Heredity, 80, 509-518. 
Table 2 Descriptive data for all mouse DA characters (DA1 = DA in C1, etc.) with significant LOD scores in the QTL analysis. $a$, additive genotypic value; $d$, dominance genotypic value; $\%$, percentage of total variation explained. Confidence intervals for the QTL locations also are given, some of which reach the proximal or distal marker at the end ( ${ }^{\text {end }}$ ) of the chromosome

\begin{tabular}{|c|c|c|c|c|c|c|c|c|}
\hline 7 & D7Mit21 & 30 & D7Mit $21+12-D 7 N d s 1+12$ & DA1 & $2.47^{*}$ & 8.4 & $-0.038 \pm 0.040^{*}$ & $-0.351 \pm 0.109 * *$ \\
\hline 12 & D12Mit37 & 0 & D12Mit $37+0^{\text {end }}-8$ & DA6 & $2.64^{*}$ & 2.5 & $-0.054 \pm 0.025^{* *}$ & $-0.094 \pm 0.035^{* *}$ \\
\hline 13 & D13Mit1 & 10 & D13Mit $1+0^{\text {end }}-28$ & DA3 & $3.28^{* *}$ & 6.7 & $0.075 \pm 0.065$ & $0.248 \pm 0.067 * *$ \\
\hline 13 & D13Mit1 & 16 & D13Mit $1+0^{\text {end }}-34$ & DASUM & $1.97^{*}$ & 8.1 & $0.020 \pm 0.023$ & $0.711 \pm 0.246^{* *}$ \\
\hline 17 & D17Mit16 & 30 & D17Mit $46+0^{\text {end }}-D 17$ Mit $16+36^{\text {end }}$ & $\mathrm{DA} 2$ & & & $0.140 \pm 0.060^{*}$ & $-0.155 \pm 0.113$ \\
\hline 18 & D18Mit17 & 18 & D18Mit $12+0^{\text {end }}-D 18 M i t 17+44^{\text {end }}$ & DA5 & $2.15^{*}$ & 4.8 & $0.082 \pm 0.039^{*}$ & $-0.151 \pm 0.064^{*}$ \\
\hline 19 & D19Mit14 & 22 & D19Mit $14+0^{\text {end }}-50^{\text {end }}$ & $\mathrm{DA} 2$ & $1.90^{*}$ & 3.8 & $-0.169 \pm 0.061^{* *}$ & $-0.117 \pm 0.111$ \\
\hline
\end{tabular}

${ }^{*} P<0.05 ; * * P<0.01$.

higher than the six expected by chance alone. Two LOD scores also reach significance at the $1 \%$ level, but one of these scores was generated by two QTLs on chromosome 17 and thus the effective number reaching significance at this level is really one, precisely as expected by chance alone. Further, one QTL on chromosome 13 (see Table 2) affects overall DA (DASUM), again as expected by chance alone. In general, therefore, there is little evidence for genuine QTLs affecting DA in these skeletal characters; instead, it is likely that at least some of these QTLs are simply artifacts of the sampling process.

Results of the interval mapping for FA in the skeletal characters (see Table 3) are somewhat different. Thus 10 QTLs significantly $(P<0.05)$ affect FA in these characters, including three at the $1 \%$ level. Further, two QTLs, one on chromosome 11 and another on chromosome 15, significantly affect FASUM. Like that for DASUM, however, these results for FASUM appear largely attributable to the significant QTLs for FA3 on these two chromosomes because the sum of all FA values except FA3 did not produce significant results in QTL runs. In any event, the number of QTLs significantly affecting FA in the characters is greater than that expected by chance alone, suggesting that some of these QTLs are genuine.

The QTLs affecting FA are found on eight of the 19 autosomes, and judging by their confidence intervals, all are typically at or near locations that include

Table 3 Descriptive data for all mouse FA characters (FA1 = FA in C1, etc.) with significant LOD scores in the QTL analysis. $a$, additive genotypic value; $d$, dominance genotypic value; $\%$, percentage of total variation explained. Confidence intervals for the QTL locations also are given, some of which reach the proximal or distal marker at the end $\left({ }^{\text {end }}\right)$ of the chromosome or were undetermined $(\mathrm{u})$

\begin{tabular}{|c|c|c|c|c|c|c|c|c|}
\hline Chrom. & Marker & $\mathrm{cM}$ & Confidence interval & Trait & LOD & $\%$ & $a$ & $d$ \\
\hline 4 & D4Mit17 & 4 & D4Mit $2+16-D 4 M i t 45+2$ & FA6 & $2.98 *$ & 3.2 & $-0.071 \pm 0.025^{* *}$ & $-0.084 \pm 0.036^{*}$ \\
\hline 7 & $D 7 N d s 1$ & 0 & D7Mit $21+24-D 7 M i t 9+16$ & FA6 & $2.36^{*}$ & 2.1 & $0.073 \pm 0.022 * *$ & $0.001 \pm 0.015$ \\
\hline 8 & D8Mit8 & 0 & $D 8 M i t 8+0^{\text {end }}-u$ & FA1 & $2.36^{*}$ & 2.3 & $0.027 \pm 0.041$ & $-0.143 \pm 0.044^{* *}$ \\
\hline 9 & D9Mit8 & 18 & D9Mit $4+10-D 9 M i t 8+44^{\text {end }}$ & FA3 & $2.59 *$ & 5.2 & $0.064 \pm 0.026^{*}$ & $0.178 \pm 0.073^{*}$ \\
\hline 9 & D9Mit8 & 28 & D9Mit8 $+6-44^{\text {end }}$ & FA4 & $1.98 *$ & 4.4 & $0.097 \pm 0.039 *$ & $-0.134 \pm 0.077$ \\
\hline 11 & D11Mit14 & 16 & D11Mit $15+12-D 11$ Mit $14+30^{\text {end }}$ & FASUM & $2.31^{*}$ & 4.2 & $0.272 \pm 0.091 * *$ & $0.203 \pm 0.161$ \\
\hline 13 & D13Mit1 & 50 & D13Mit1 + 10-D13Mit19+14 & FA3 & $2.87 * *$ & 3.2 & $-0.097 \pm 0.027 * *$ & $0.069 \pm 0.090$ \\
\hline 15 & D15Mit13 & 6 & D15Mit13 $+0^{\text {end }}-16$ & FA3 & $2.89 * *$ & 3.8 & $0.026 \pm 0.026$ & $-0.168 \pm 0.048 * *$ \\
\hline 15 & D15Mit13 & 8 & D15Mit13 + 0-D15Mit15 + 12 & FASUM & $1.96^{*}$ & 3.3 & $0.184 \pm 0.075^{*}$ & $-0.293 \pm 0.169$ \\
\hline 17 & D17Mit46 & 0 & D17Mit $46+0^{\text {end }}-8$ & FA1 & $2.12^{*}$ & 2.2 & $0.075 \pm 0.033^{*}$ & $-0.100 \pm 0.046^{*}$ \\
\hline
\end{tabular}

${ }^{*} P<0.05 ; * * P<0.01$.

(C) The Genetical Society of Great Britain, Heredity, 80, 509-518. 
QTLs affecting the mean of the sides or DA in one of the skeletal characters (see Fig. 2). Except for three instances involving QTLs on chromosomes 4, 7 and 8 affecting $\mathrm{FA}$ in $\mathrm{C} 1$ and $\mathrm{C} 6$, however, all other QTLs for FA in a given character appear to exist at locations for QTLs affecting other characters (or DA in other characters). The mean effect of these QTLs is $3.9 \%$, a value very comparable to that for QTLs affecting DA and the overall characters. However, for these QTLs affecting FA in the individual characters (excluding FASUM), the mean absolute dominance genotypic value of $0.116 \pm 0.019$ is significantly greater (1-tailed $t=1.95 ; P<0.05$ ) than the mean absolute additive genotype value of $0.072 \pm 0.009$. For the 10 significant QTLs also, eight $a$-values and seven $d$-values reach statistical significance $(P<0.05)$. Further, five of the seven significant $d$-values are negative in sign (including all three that reach significance at the $1 \%$ level), indicating less asymmetry in the heterozygotes than expected for a purely additive model.

\section{Discussion}

It was somewhat surprising that the number of QTLs significantly affecting DA in the discrete skeletal characters was no greater than that expected by chance, especially given that the opposite was true for 10 different continuously varying mandible characters measured in these same mice (Leamy et al., 1997). It might be thought that this should have been expected given the general lack of overall DA in the characters used here, but, theoretically, different alleles of a QTL could act to produce DA in opposite directions, possibly cancelling each other and producing no significant overall DA. This was seen in fact in the previous QTL study where the only two mandible characters showing no overall DA were significantly affected by five of the 16 total QTLs found for DA in these characters (Leamy et al., 1997). However, DA in metoptic roots abnormal (C6), the only character used in this study that did show overall DA, was significantly affected by only one QTL, as is expected by chance alone. Contrary to what has been assumed for most bilateral characters (Palmer \& Strobeck, 1992), therefore, there is little evidence of a genetical basis for DA in the skeletal characters used here.

On the other hand, there seemed to be sufficient evidence from the probability arguments given previously for a significant number of QTLs affecting FA in these characters. Central to these probability arguments was the assumption that the characters are independent, and this was verified by the nonsignificant pairwise correlations of FA in each of the six characters. It might be argued that metoptic roots abnormal (C6) should be omitted from the probability calculations above because it exhibited DA and thus not 'pure' FA, but only two QTLs significantly $(P<0.01)$ affected FA in this character (see Table 3) and the remaining eight QTLs would still be above the $5 \times 0.05 \times 19=4.75$ expected by chance alone to affect FA in characters C1-C5. All this implies that there are genuine QTLs affecting FA in the skeletal characters and thus that their variation is not all environmental in origin.

The genetical basis for FA in these characters, however, is weak. Although QTLs for FA contributed on average $3.9 \%$ of the total variation, these QTLs were so few that they generated a cumulative effect that ranged only from $0 \%$ (FA in maxillary foramen II, C2) to $15.3 \%$ (FA in foramen palatinus minus posterior, C3). The total genetical variance, or broad-sense heritability (Falconer, 1989), averages 0.065 for FA in these characters. Although low, this value is similar to the mean (narrow-sense) heritability estimate of 0.04 calculated for FA in eight different discrete skeletal characters in random-bred mice (Leamy, 1997b). Given the standard errors typically associated with heritability estimates (Falconer, 1989), it is not surprising that heritabilities of FA in bilateral characters that are this low have failed to reach significance in many studies (Leamy, 1997a,b).

Dominance may be important in the action of the QTLs significantly affecting FA in the skeletal characters. The average of the absolute $d$-values $(0.116 \pm 0.019 ; 0.134$ if $\mathrm{C} 6$ is omitted) for these QTLs was significantly higher than the average of the absolute $a$-values $(0.072 \pm 0.009$ with C6 omitted or retained). This also was the case for the QTLs affecting FA in the mandible characters in these mice (Leamy et al., 1997) where the disparity between the mean $d$ - $(0.021 \pm 0.004)$ and $a$-values $(0.008 \pm 0.001)$ was even greater. However, dominance is harder to detect than additivity in these models (Leamy et al., 1997), so it is difficult to know how much significance to attach to this result. Nonetheless, these results do not refute the generally held belief that dominance effects play important roles in the production of FA in bilateral characters (Livshits \& Kobyliansky, 1991).

It was interesting that five of the seven significant $d$-values for QTLs affecting FA were negative in sign. This suggests that heterozygotes for these five QTLs tend to be less asymmetrical than homozygotes, indicating that heterozygotes are more

(c) The Genetical Society of Great Britain, Heredity, 80, 509-518. 
developmentally stable for these characters than homozygotes. Overall heterozygosity does not vary much between $F_{2}$ animals, so the genetic effects mapping to individual chromosomal regions identified here are likely to result from heterozygosity at specific loci rather than over the genome as a whole. These QTLs control phenotypic variability in character states rather than the character states themselves. It is possible that environmental effects are responsible for the FA detected here but that genotypes at specific loci make the phenotype more, or less, susceptible to environmental perturbations.

QTLs for a given individual skeletal character (say C3) generally did not map in the same region as the QTL for FA in that same character, which is consistent with the results from a previous mandible FA study (Leamy et al., 1997). There were three exceptions to this, but two of these involved QTLs for C6 that may simply also have reached significance for FA in this character because of its large amount of asymmetry. If $\mathrm{C} 6$ is omitted from consideration as before, then seven of the remaining eight QTLs for FA in the skeletal characters are not at the same location as QTLs affecting those specific characters (see Fig. 2). Perhaps this kind of result should not have been surprising, for some years ago Caligari \& Mather (1975) discovered that genes affecting the response in chaeta number in Drosophila to environmental (temperature) change were on a different chromosome from genes directly affecting chaeta number.

If we are truly to think of these QTLs as genes that influence the level of FA and hence developmental stability in the $F_{2}$ mice, then what might be their nature? Although this is presently unknown, it seemed useful to search for possible candidates for these QTLs from the map positions of genes given in the Mouse Genome Database (1995). This was previously carried out for the QTLs identified for both DA and FA in the mandible characters in these mice, and several of these QTLs were found to map close to genes responsible for the production of growth factors, hormones, and hormone receptors (Leamy et al., 1997). A search for likely candidates at or near the positions for the QTLs on chromosomes 9, 11 and 15 influencing FA in the skeletal characters showed that they mapped close to the acetylcholine receptor alpha three neural (Acra3) locus, the growth hormone $(G h)$ locus and the growth hormone receptor $(G h r)$ locus. The similarity of locations especially of the two QTLs on chromosomes 11 and 15 to those previously identified as affecting FA in the mandible characters (Leamy et al., 1997) bolsters the likelihood that genes controlling hormones and their receptors may also influence FA. This finding, however, begs for additional QTL investigations that would discover whether these sorts of genes influence FA in other bilateral characters as well.

\section{Acknow ledgements}

It is a pleasure to thank Shemelis Beyene, Marguerite Butler, Eirik Cheverud, Duncan Irshick and Natalia Vasey for help with laboratory work, Glenn Conroy for the use of microscope equipment, Jeffrey Baliff for scoring the skeletal characters, and two anonymous reviewers for useful revision suggestions. This research was supported in part by funds provided by The University of North Carolina at Charlotte and by National Science Foundation grants BSR-9106565 and DEB-9419992.

\section{References}

BERRY, R. J. 1963. Epigenetic polymorphism in wild populations of Mus musculus. Genet. Res., 4, 193-220.

CAligari, P. D. S. AND MATHER, K. 1975. Genotypeenvironment interaction: III. Interactions in Drosophila melanogaster. Proc. R. Soc. B., 191, 387-411.

CHEVERUd, J. M., ROUTMAN, E. J., DUARTE, F. A. M., SWINDEREN, B. V., COTHRAN, K. AND PEREL, C. 1996. Quantitative trait loci for murine growth. Genetics, 142, $1305-1319$.

DIETRICH, W. F., MILlER, J., STEEN, R., MERCHANT, M., DAMron-BOles, D., HUSAIN, Z. ET AL. 1996. A comprehensive genetic map of the mouse genome. Nature, 380, 149-152.

FALCONER, D. S. 1989. Introduction to Quantitative Genetics, 3rd edn. John Wiley and Sons, New York.

Goodale, H. 1941. Progress report on possibilities in progeny test breeding. Science, 94, 442-443.

HALEY, C. S. AND KNOTT, S. A. 1992. A simple regression method for mapping quantitative trait loci in line crosses using flanking markers. Heredity, 69, 315-324.

LANDER, E. S. AND BOTSTEIN, D. 1989. Mapping Mendelian factors underlying quantitative traits using RFLP linkage maps. Genetics, 121, 185-199.

LANDER, E. S., GREEN, P., ABRAHAMSON, J., BARLOW, A., DALEY, M., LINCOLN, S. ET AL. 1987. MAPMAKER, an interactive computer package for constructing primary genetic linkage maps of experimental and natural populations. Genomics, 1, 174-181.

LEAMY, L. 1984. Morphometric studies in inbred and hybrid house mice. V. Directional and fluctuating asymmetry. Am. Nat., 123, 579-593.

LEAMY, L. 1997a. Is developmental stability heritable? $J$. Evol. Biol., 10, 21-29. 
LEAMY, L. 1997b. Genetic analysis of fluctuating asymmetry for skeletal characters in mice. J. Hered., 88, $85-92$.

LEAMY, L. J., ROUtMAN, E. J. AND CHEVERud, J. M. 1997. A search for quantitative trait loci affecting asymmetry of mandibular characters in mice. Evolution, 51, 957-969.

LINCOLN, S., DALY, M. AND LANDER, E. 1992. Constructing genetic maps with MAPMAKER/EXP 3.0., Technical Report, 3rd edn. Whitehead Institute, Cambridge, MA.

LIVSHITS, G. AND KOBYLIANSKY, E. 1991. Fluctuating asymmetry as a possible measure of developmental homeostasis in humans. Hum. Biol., 63, 441-466.

MACARTHur, J. 1944. Genetics of body size and related characters. I. Selection of small and large races of the laboratory mouse. Am. Nat., 78, 142-157.

MOUSE GENOME DATABASE (MGD). 1995. Mouse Genome Informatics Project. The Jackson Laboratory, Bar Harbor, ME. World Wide Web (URL: http: //www.informatics.jax.org).

PALMER, A. R. 1994. Fluctuating asymmetry analyses: a primer. In: Markow, T. A. (ed.) Developmental Instability: Its Origins and Evolutionary Implications, pp. 335-364. Kluwer, Dordrecht.

PALMER, A. R. AND STROBECK, C. 1992. Fluctuating asymmetry as a measure of developmental stability: implications of non-normal distributions and power of statistical tests. Acta Zool. Fenn., 191, 57-72.
PALMER, A. R., STROBECK, C. AND CHIPPINDALE, A. K. 1994. Bilateral variation and the evolutionary origin of macroscopic asymmetries. In: Markow, T. A. (ed.) Developmental Instability: Its Origins and Evolutionary Implications, pp. 203-220. Kluwer, Dordrecht.

RICE, w. R. 1989. Analyzing tables of statistical tests. Evolution, 43, 223-225.

Routman, E. AND CHeVerud, J. 1995. Polymorphism for PCR-analysed microsatellites: data for two additional mouse strains and the utility of agarose gel electrophoresis. Mammal. Genome, 6, 401-404.

SOKAL, R. R. AND ROHLF, J. F. 1995. Biometry, 3rd edn. Freeman, San Francisco.

THORNHILL, R. AND SAUER, K. P. 1992. Genetic sire effects on the fighting ability of sons and daughters and mating success of sons in the scorpionfly (Panorpa vulgaris). Anim. Behav., 43, 255-264.

TRUSLOVE, G. M. 1954. Genetical studies on the skeleton of the mouse. XIII. Variations in the presphenoid. $J$. Genet., 52, 589-602.

ZAKHAROV, v. M. 1994. Appearance, fixation and stabilisation of environmentally induced phenotypic changes as a microevolutionary event. In: Markow, T. A. (ed.) Developmental Instability: Its Origins and Evolutionary Implications, pp. 229-236. Kluwer, Dordrecht.

ZENG, Z. B. 1994. Precision mapping of quantitative trait loci. Genetics, 136, 1457-1468.

Appendix Descriptive data for all mouse discrete skeletal characters with significant LOD scores in the QTL analysis. $a$, additive genotypic value; $d$, dominance genotypic value; $\%$, percentage of total variation explained. Confidence intervals for the QTL locations are also given, some of which reach the proximal or distal marker at the end $\left({ }^{\text {end }}\right)$ of the chromosome or were undetermined $(\mathrm{u})$

\begin{tabular}{|c|c|c|c|c|c|c|c|c|}
\hline Chrom. & Marker & $\mathrm{cM}$ & Confidence interval & Trait & LOD & $\%$ & $a$ & $d$ \\
\hline 1 & D1Mit14 & 18 & $D 1 M i t 7+10-D 1 M i t 14+36^{\text {end }}$ & $\mathrm{C} 5$ & $3.62 * *$ & 6.2 & $-0.160 \pm 0.040^{* *}$ & $-0.056 \pm 0.065$ \\
\hline 2 & D2Mit28 & 12 & $D 2 M i t 17+2-D 2 M i t 28+12^{\text {end }}$ & $\mathrm{C} 4$ & $2.05^{*}$ & 3.0 & $-0.035 \pm 0.020$ & $-0.103 \pm 0.041^{*}$ \\
\hline 2 & D2Mit17 & 2 & D2Mit $17+0^{\text {end }}-D 2 M i t 28+12^{\text {end }}$ & $\mathrm{C} 5$ & $1.77^{*}$ & 1.9 & $0.002 \pm 0.006$ & $-0.130 \pm 0.046^{* *}$ \\
\hline 4 & D4Mit17 & 4 & D4Mit $2+20-D 4 M i t 16+8$ & $\mathrm{C} 1$ & $2.51^{*}$ & 2.9 & $0.078 \pm 0.033^{*}$ & $-0.121 \pm 0.050^{*}$ \\
\hline 4 & D4Mit17 & 6 & D4Mit $2+18-D 4 M i t 45+2$ & C6 & $4.97 * *$ & 5.4 & $-0.114 \pm 0.028^{* *}$ & $-0.102 \pm 0.040^{* *}$ \\
\hline 6 & $D 6 N d s 5$ & 26 & $D 6 N d s 5+22-26^{\text {end }}$ & C5 & $6.65 * *$ & 6.9 & $0.165 \pm 0.031^{* *}$ & $-0.074 \pm 0.045$ \\
\hline 7 & D7Mit17 & 4 & D7Nds1 $+4-D 7 M i t 17+10$ & $\mathrm{C} 2$ & $2.82 * *$ & 3.3 & $0.051 \pm 0.032$ & $0.131 \pm 0.041^{* *}$ \\
\hline 7 & $D 7 N d s 1$ & 6 & D7Mit $21+46-D 7 M i t 17+12$ & $\mathrm{C} 5$ & $4.70^{* *}$ & 5.1 & $0.131 \pm 0.032 * *$ & $0.106 \pm 0.048^{*}$ \\
\hline 7 & $D 7 N d s 1$ & 0 & $D 7 M i t 21+44-D 7 M i t 17+0$ & C6 & $2.25^{*}$ & 2.1 & $0.074 \pm 0.025^{* *}$ & $0.046 \pm 0.036$ \\
\hline 8 & D8Mit8 & 0 & D8Mit $8+0^{\text {end }}-u$ & $\mathrm{C} 1$ & $5.03 * *$ & 4.7 & $-0.026 \pm 0.024$ & $-0.205 \pm 0.044^{* *}$ \\
\hline 8 & D8Mit8 & 0 & $D 8 M i t 8+0^{\text {end }}-u$ & $\mathrm{C} 2$ & $2.10^{*}$ & 1.9 & $0.010 \pm 0.020$ & $0.112 \pm 0.037^{* *}$ \\
\hline 8 & D8Mit8 & 82 & $D 8 M i t 8+u-82$ & $\mathrm{C} 5$ & $2.11^{*}$ & 2.3 & $0.066 \pm 0.031 *$ & $0.107 \pm 0.043^{*}$ \\
\hline 11 & D11Mit14 & 16 & D11Mit $14+0-30^{\text {end }}$ & $\mathrm{C} 2$ & $3.28^{* *}$ & 6.5 & $0.085 \pm 0.038^{*}$ & $0.171 \pm 0.054^{* *}$ \\
\hline 11 & D11Mit15 & 4 & D11Mit64+4-D11Mit15+16 & $\mathrm{C} 4$ & $2.77 *$ & 3.2 & $-0.050 \pm 0.023^{*}$ & $0.093 \pm 0.030^{* *}$ \\
\hline 11 & D11Mit15 & 14 & D11Mit64+6-D11Mit14+24 & $\mathrm{C} 5$ & $2.78^{*}$ & 3.3 & $0.118 \pm 0.033^{* *}$ & $-0.021 \pm 0.054$ \\
\hline 14 & D14Mit5 & 18 & D14Mit5 $+8-18^{\text {end }}$ & C6 & $2.82 * *$ & 2.8 & $-0.083 \pm 0.025^{* *}$ & $0.056 \pm 0.039$ \\
\hline 15 & D15Mit5 & 10 & D15Mit13+0-D15Mit5 +8 & $\mathrm{C} 5$ & $2.74 * *$ & 3.6 & $0.122 \pm 0.035^{* *}$ & $-0.040 \pm 0.019^{*}$ \\
\hline 19 & D19Mit14 & 50 & D19Mit14 $+26-50^{\text {end }}$ & $\mathrm{C} 1$ & $2.10^{*}$ & 2.2 & $0.086 \pm 0.031 * *$ & $-0.063 \pm 0.042$ \\
\hline
\end{tabular}

$* P<0.05 ; * * P<0.01$ 